\title{
ASPECTOS HEMODINÂMICOS DA CIRCULAÇÃO EXTRACORPÓREA EM CÃES
}

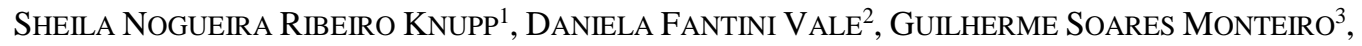 \\ MÔNICA JORGE LUZ ${ }^{2}$, JusSARA PETERS SHEFFER ${ }^{2}$, ANDRÉ LACERDA OLIVEIRA ${ }^{4}$ \\ ${ }^{1}$ Pós Graduanda da universidade Federal de Campina Grande, Patos, PB, Brasil - sheilanribeiro@ hotmail.com \\ ${ }^{2}$ Pós-graduandas da Universidade Estadual do Norte Fluminense, Campos de Goytacazes, RJ, Brasil. \\ ${ }^{3}$ Veterinário, Mestre, Rio de Janeiro, RJ, Brasil. \\ ${ }^{4}$ Professor Doutor da Universidade Estadual do Norte Fluminense, Campos de Goytacazes, RJ, Brasil.
}

\section{RESUMO}

A circulação extracorpórea (CEC) promove alterações graves no organismo do paciente que necessita ser submetido a esse procedimento, que pode, no entanto, ser contornadas ou minimizadas com manobras específicas para cada situação. Apesar de ser uma técnica já estabelecida na medicina humana, ainda existem avanços a serem alcançados. Objetivou-se com este trabalho descrever a técnica de circulação extracorpórea e informar seus efeitos hemodinâmicos quando aplicada em cães. Utilizaram-se quatro cães hígidos, sem raça definida. Os animais foram submetidos à anestesia e monitoramentos e coletas sanguíneas foram realizadas (T0). Em seguida, os animais foram submetidos à esternotomia mediana, canulação da artéria aorta e veias cava cranial e caudal e mantidos por um período de 30 minutos em CEC (T1), foram, depois, desconectados da máquina de $\mathrm{CEC}$, permanecendo por 30 minutos em processo de reperfusão (T2), seguidos de uma hora de reperfusão (T3), sendo, então, eutanasiados. Avaliaram-se os seguintes parâmetros: pressão arterial média, pressão venosa central, oxigenação $\left(\mathrm{SaO}_{2}\right)$ e capnografia $\left(\mathrm{ETCO}_{2}\right)$. PAM, PVC e $\mathrm{SaO}_{2}$ permaneceram dentro da normalidade durante os tempos avaliados, já a média observada do $\mathrm{ETCO}_{2}$ estava abaixo apenas em um dos animais experimentais, porém os valores mantiveram-se dentro da normalidade na maioria dos animais experimentais. Concluiu-se que a CEC é possível de ser realizada em cães, sem prejuízos hemodinâmicos graves, considerando-se os parâmetros avaliados neste experimento.

PALAVRAS-CHAVE: Cães, circulação extracorpórea, cardiologia veterinária, cirurgia.

\section{HEMODYNAMIC ASPECTS OF CARDIOPULMONARY BYPASS IN DOGS}

\section{ABSTRACT}

The cardiopulmonary bypass (CPB) promotes major changes to the body of the patient that needs to be subjected to this procedure, but it can be circumvented or minimized with specific maneuvers for each situation. Despite being an established technique in human medicine, there are still improvements to be achieved. The purposes of this work were to clarify the cardiopulmonary bypass and inform its hemodynamic effects when applied to dogs. Four mongrel healthy dogs were used. The animals were anesthetized and monitored and samples were collected (T0). After that, the animals underwent median sternotomy and cannulation of the aorta and cranial and caudal vena cava and they were kept in CPB for a period of 30 minutes (T1), then disconnected from the CPB machine during 30 minutes in reperfusion process (T2), followed by one hour of reperfusion (T3), and then they were euthanized. The following parameters were evaluated: mean arterial pressure (MAP), central venous pressure (CVP), oxygenation $\left(\mathrm{SaO}_{2}\right)$ and capnography $\left(\mathrm{ETCO}_{2}\right)$. MAP, CVP and $\mathrm{SaO}_{2}$ remained within normal limits during the times evaluated. Although the observed average of $\mathrm{ETCO}_{2}$ was low in one of the experimental 
animals, it maintained the values within the normal range in most experimental animals. We concluded that CPB can be performed in dogs without severe hemodynamic compromise, considering the parameters evaluated in this experiment.

KEYWORDS Cardiopulmonary bypass, dog, surgery, veterinary cardiology.

\section{INTRODUÇÃO}

A cirurgia torácica está se tornando cada vez mais uma realidade em hospitais veterinários, principalmente devido aos avanços tecnológicos e à crescente capacitação técnica dos médicos veterinários. Já foram relatadas na literatura veterinária algumas intervenções cirúrgicas com resultados satisfatórios visando à correção da tetralogia de Fallot $^{1}$, correção da persistência do ducto arterioso $^{2}$ e correção de comunicação interatrial ${ }^{3}$. Entretanto, cirurgias intracardíacas que exigem maior tempo transoperatório, como a correção da comunicação interventricular, estenose pulmonar, ou estenose subaórtica e algumas valvuloplastias, além do transplante cardíaco, só podem ser realizadas mantendo-se o paciente em circulação extracorpórea (CEC), pois o cirurgião necessita intervir sem perda excessiva de sangue e por um período de tempo superior aos cinco minutos estabelecidos pela inflow occlusion. Além desse fator, outra vantagem da CEC é a manutenção do coração parado e vazio durante todo o ato cirúrgico, facilitando o procedimento.

No circuito básico da perfusão, o sangue é drenado do átrio direito (uma cânula) ou das veias cava cranial e caudal (duas cânulas), por gravidade e sifonagem, para o reservatório venoso dos oxigenadores de membranas. Nos oxigenadores de membranas, o sangue do reservatório venoso é impulsionado pela bomba arterial através do compartimento das membranas onde, por difusão, capta o oxigênio e elimina o dióxido de carbono. Da saída arterial do oxigenador de membranas, o sangue continua o seu percurso pela linha arterial, até a cânula introduzida na aorta ascendente ou, opcionalmente, em outro ponto do sistema arterial, como a artéria femoral ${ }^{4}$. A reinfusão do sangue é preferencialmente realizada na aorta ascendente, porém a artéria femoral pode ser empregada em casos de re-operações, situações de emergência, instalação de oxigenação assistida, aneurismas ou dissecções de aorta, sendo utilizadas cânulas diferenciadas e específicas para cada acesso ${ }^{5}$.

O circuito básico também é composto pelas linhas aspiradoras, que servem para carrear o sangue extravasado das cavidades cardíacas, por meio das bombas aspiradoras, para o reservatório de cardiotomia, mantendo-se constante o volume de sangue do paciente ${ }^{4}$. Esta é uma ferramenta fundamental para a manutenção da pressão e volemia do paciente, um dos principais aspectos a serem controlados durante a CEC.

A aplicação da CEC na rotina médica veterinária ainda não foi alcançada com êxito no Brasil por ser um procedimento oneroso e ocasionar alta mortalidade ${ }^{6}$, além da necessitar de uma grande equipe capacitada na área. Apesar de ser uma técnica já estabelecida e amplamente aplicada na medicina humana ${ }^{7}$, ainda existem avanços a serem alcançados em ambas as áreas, sendo que na medicina veterinária precisa ser aperfeiçoada ou adaptada a fim de reduzir a mortalidade ao máximo. Objetivou-se com este trabalho descrever a técnica de circulação extracorpórea e seus efeitos hemodinâmicos, quando aplicada em cães, considerando a pressão arterial média, pressão venosa central, saturação arterial de oxigênio e a concentração de $\mathrm{CO}_{2}$ ao final da expiração.

\section{MATERIAL E MÉTODOS}

O experimento foi submetido ao Comitê de Ética em Experimentação Animal da Universidade Estadual Norte Fluminense Darcy Ribeiro, sob o número de registro 25. Foram utilizados quatro cães domésticos (Canis lupus familiaris), sem raça definida, adultos, com peso entre 15 a 25 kilogramas $(\mathrm{kg})$, selecionados de forma aleatória. Quando admitidos no hospital veterinário, foram submetidos a exames clínicos e laboratoriais (hemograma, avaliação de albumina, análise de enzimas hepáticas e avaliação renal), além de eletrocardiograma (ECG), a fim de detectar quaisquer alterações que pudesse impedi-los de participar do estudo.

Anteriormente ao procedimento cirúrgico, os animais foram mantidos em jejum alimentar de 12 horas e hídrico de oito horas. Cada animal recebeu medicação pré-anestésica com maleato de acepromazina, na dose de $0,1 \mathrm{mg} \cdot \mathrm{kg}^{-1}$, associado à morfina, na dose de $0,2 \mathrm{mg} \cdot \mathrm{kg}^{-1}$, pela via muscular (IM). Após 10 minutos, realizou-se tricotomia ampla da região ventral do tórax, assim como da 
face cranial dos membros torácicos, para posterior cateterização da veia cefálica e jugular, e tricotomia da região cervical ventral e medial dos membros pélvicos, para cateterização da artéria femoral.

Após a indução anestésica com proporfol (5 $\left.\mathrm{mg} \cdot \mathrm{kg}^{-1}\right)$, o paciente foi intubado e a sonda traqueal acoplada ao aparelho de anestesia inalatória, em sistema fechado, mantendo-se a inalação de isoflurano a aproximadamente 0,5 CAM $(1,0 \%)$ e ventilação provida manualmente por balão acoplado ao aparelho de anestesia.

Depois da antissepsia da região medial dos membros pélvicos, foi estabelecida uma linha arterial com dissecção da artéria femoral e sua punção com cateter que, em seguida, foi conectado a um equipo de soro e a um manômetro, mantendose, dessa forma, a pressão arterial média (PAM) monitorada continuamente (Figura 1). Os períodos imediatamente anteriores ao procedimento cirúrgico (T0), logo após o término da CEC (T1), após 30 minutos de reperfusão pós-CEC (T2) e após uma hora de reperfusão pós-CEC (T3) foram considerados para avaliação estatística.

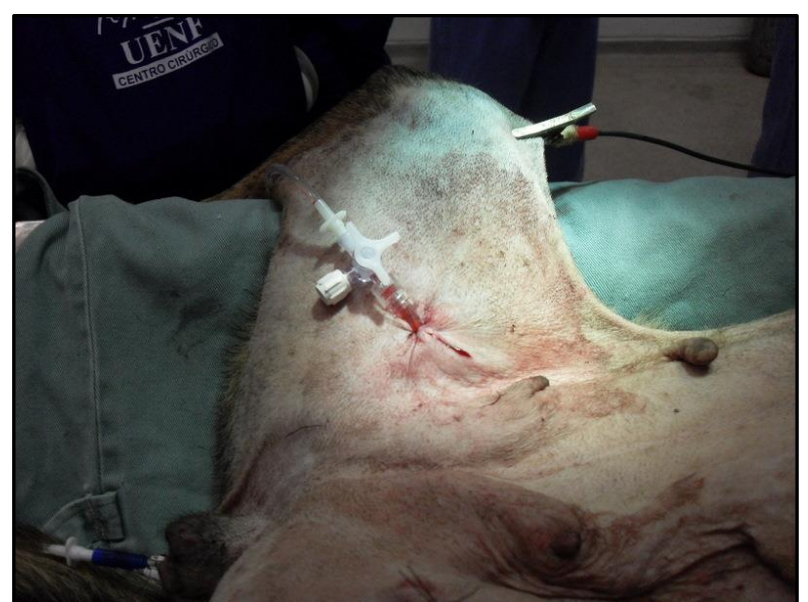

Figura 1. Artéria femoral de um cão canulada e conectada a uma torneira de três vias, equipo de soro e manômetro, permitindo monitoramento constante da pressão arterial média invasiva e intervenções, quando necessárias, para sua manutenção em níveis adequados.

A cateterização venosa central ocorreu de forma percutânea, com um cateter de poliuretano de lume duplo (Figura 2), conectado a um equipo de soro (formando coluna de água), mantendo-se, dessa forma, monitoramento contínuo da pressão venosa central (PVC), sendo considerados para avaliação estatística os mesmos tempos utilizados na avaliação da PAM.

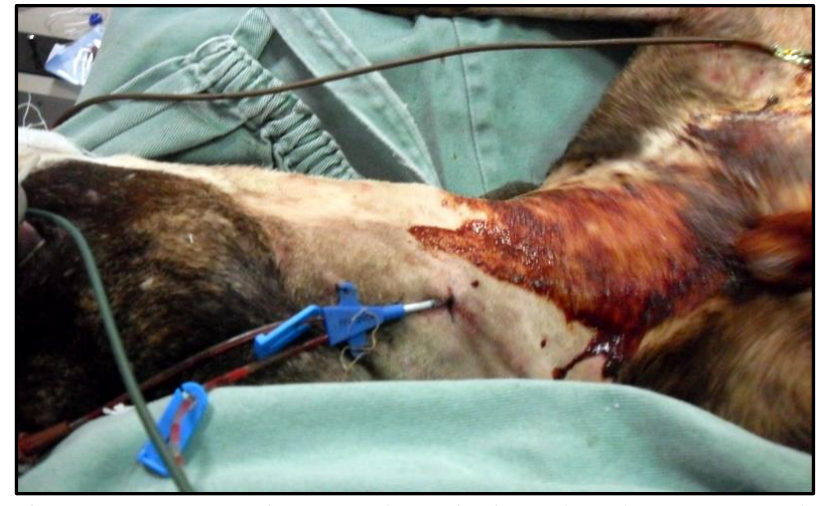

Figura 2. Cateterização da veia jugular de um cão, de forma percutânea, com um cateter de poliuretano de lume duplo, fixado à pele com fio de nylon 2-0, conectado a um equipo de soro, mantendo-se monitoramento contínuo da pressão venosa central (PVC), permitindo intervenções, quando necessárias, para sua manutenção em níveis adequados.

A oximetria de pulso permitiu o monitoramento da saturação de oxigênio, com sensor mantido na língua do animal, através do Monitor Multiparamétrico Adivisor ${ }^{\circledR}$ (Smith Medical PM), sendo considerados para avaliação estatística os mesmos tempos utilizados na avaliação da PAM.

Ao término do preparo do animal e após a estabilização da anestesia, os cães foram mantidos em decúbito dorsal para realização da esternotomia mediana. A incisão iniciou-se na linha média torácica, por toda a extensão do esterno, incidindo sobre pele, subcutâneo e musculatura com bisturi elétrico monopolar (Figura 3).

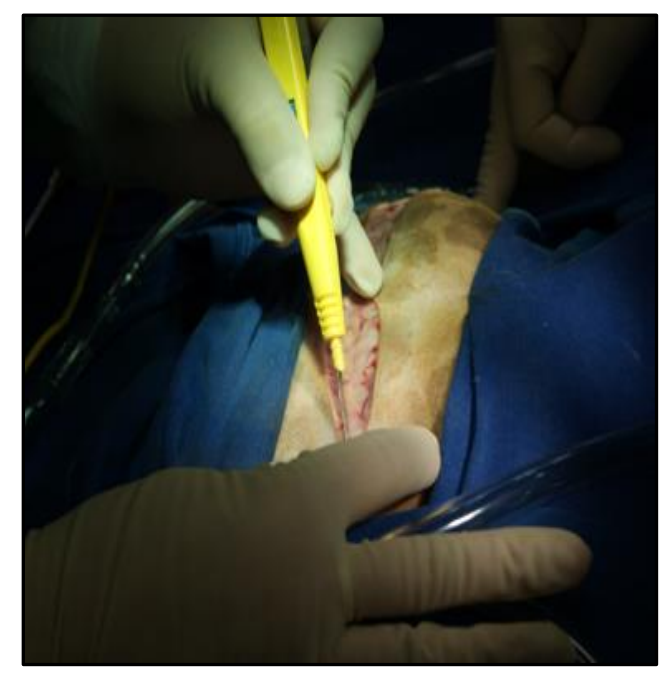

Figura 3. Acesso intratorácico, incidindo-se na linha média torácica do cão, por toda a extensão do esterno, sobre pele, subcutâneo e musculatura, com bisturi elétrico monopolar realizando-se a incisão dos tecidos e cauterização dos vasos, evitando-se hemorragias. 
Utilizou-se também um costótomo adaptado para acesso torácico, com exposição do mediastino anterior, abertura do saco pericárdico com sua fixação às paredes torácicas (Figura 4), análise das estruturas cardíacas externas e acesso à aorta ascendente, veias cavas cranial e caudal (aurícula direita), seguindo a sequência descrita. A canulação foi realizada a partir de uma incisão longitudinal na parede dos vasos, no interior de suturas em bolsa de fumo, seguida pela introdução das cânulas e fixação das mesmas com a sutura em bolsa (Figura 5).

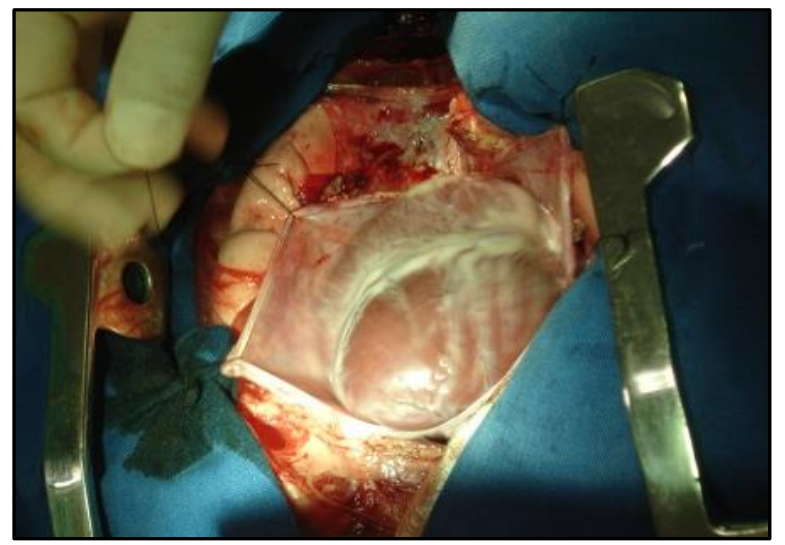

Figura 4. Preparação para o acesso aos grandes vasos cardíacos caninos, com exposição do mediastino anterior e abertura do saco pericárdico, fixando-o às paredes torácicas, facilitando o acesso e promovendo o isolamento cardíaco.

Em seguida, para melhor perfundir o miocárdio com a solução de cardioplegia, foi mantido um clamp na aorta durante 20 minutos, sendo a proteção miocárdica realizada de forma anterógrada intermitente e com cardioplegia hipercalêmica. Todos os animais foram submetidos à circulação extracorpórea utilizando-se a máquina de circulação extracorpórea e oxigenador de membrana pediátrico (Figura 6), por um período de 30 minutos.

$\mathrm{O}$ circuito de CEC foi previamente preenchido com solução de Ringer lactato, manitol $\left(0,5 \mathrm{mg} \cdot \mathrm{kg}^{-1}\right)$ a $20 \%$, heparina sódica $\left(1000\right.$ UI.L ${ }^{-1} \mathrm{de}$ prime) e bicarbonato de sódio ( $15 \mathrm{mg} . \mathrm{L}^{-1}$ de prime). A CEC foi realizada em normotermia, com fluxo arterial não pulsátil de 2,4 L.min.m ${ }^{2}$ e pressão de perfusão mantida entre 60 e $80 \mathrm{mmHg}$.

Quando necessário, foi adicionado um maior volume de heparina até que se alcançasse um tempo de coagulação ativado (TCA) acima de 400 a 480 segundos (seg). A neutralização da heparina foi obtida com sulfato de protamina, na proporção de 1:1, em relação à massa da heparina administrada durante todo o procedimento, podendo ser acrescentado até mais um terço do valor total, de acordo com o TCA.

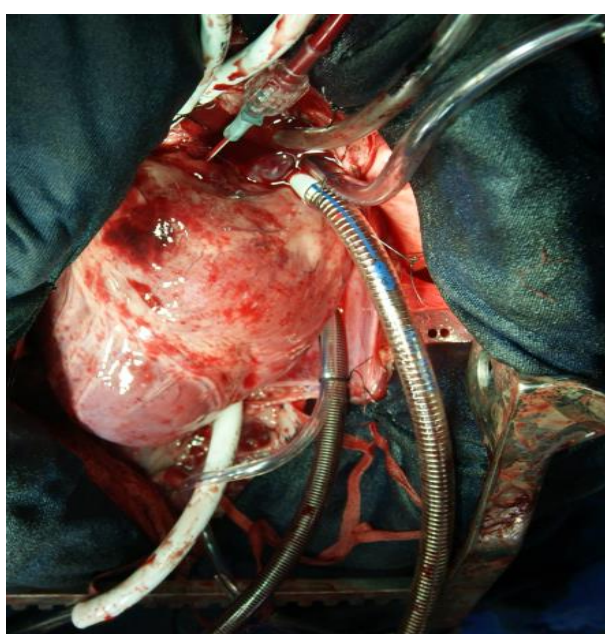

Figura 5. Canulação da artéria aorta, veia cava cranial e veia cava caudal para a realização da circulação extracorpórea (CEC) em cão.

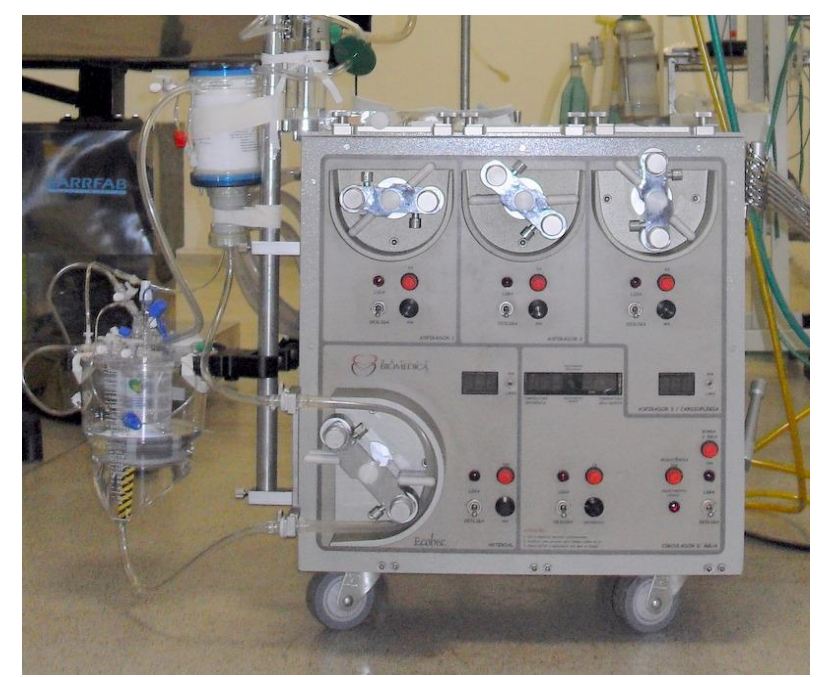

Figura 6. Máquina de circulação extracorpórea tipo roller-pump - Braile Biomédica; podem-se observar bombas de rolete, reservatório venoso e oxigenador de membranas pediátrico.

Ao final de uma hora de reperfusão pós-CEC, os animais eram eutanasiados com administração de tiopental sódico, na dose de $40 \mathrm{mg} \cdot \mathrm{kg}^{-1} \mathrm{IV}$, e cloreto de potássio, na dose de $100 \mathrm{mg} \cdot \mathrm{kg}^{-1} \mathrm{IV}$, respeitando-se os princípios éticos do uso de animais de experimentação.

Os dados obtidos foram formatados e avaliados através de análise de variância (ANOVA) para amostras paramétricas repetidas (repeated measures) e não repetidas (one way ANOVA) e, em seguida, processados no software Graph Prism 5.0, realizando-se análise de variância e teste de médias por Newman-keuls com $95 \%$ de confiabilidade e resultados expressos em média \pm erro padrão médio. 


\section{RESULTADOS E DISCUSSÃO}

A técnica utilizada para a implantação da CEC em cães mostrou-se segura e de fácil execução, apesar de ter ocorrido um acidente durante a execução da técnica no primeiro cão, com ruptura da aorta, durante a feitura da bolsa de fumo. Esse é um acidente comum de ocorrer devido à característica do endotélio e à inexperiência do cirurgião, tendendo à não reocorrência conforme maior a prática, como observado também em outro experimento ${ }^{8}$.

A hemorragia decorrente da ruptura da aorta no animal 1 foi solucionada rapidamente com a sutura da mesma, porém impossibilitou a efetivação da linha arterial através da aorta, procedendo-se, então, à técnica com a canulação da artéria femoral, assim como realizado em outros experimentos ${ }^{9-11}$, mantendo-se o animal durante 30 minutos em CEC e uma hora de reperfusão pós-CEC, assim como os demais animais, os quais não sofreram acidentes trans-cirúrgicos durante a efetivação da técnica, que foi realizada com destreza.

A pressão arterial média (PAM) permaneceu dentro da normalidade durante os tempos avaliados (Tabela 1). Isto é explicado, entre T0 e T1, pela manutenção da volemia adequada para os animais experimentais e, entre T1 e T2, pela utilização da máquina de CEC. A hipotensão arterial é relatada como de comum ocorrência durante as manobras para a canulação da aorta e das veias cavas, causada, em geral, pelo sangramento ou manipulação do coração; no entanto, é senso comum que, se o período de hipotensão arterial for curto e a entrada em perfusão for iminente, não há necessidade de intervenção, pois a circulação logo estará a cargo da $\mathrm{CEC}^{12}$ que, através do aumento das circunvoluções do rolete arterial, proporciona aumento da pressão arterial, assim como com a redução das circunvoluções produz queda da PAM, mantendo-a dentro dos valores adequados para a espécie, como no presente experimento.

É comum a PAM sofrer oscilações em consequência das alterações do tônus arteriolar, da eliminação de líquidos e da redistribuição da volemia com passagem de líquidos para o espaço intersticial ${ }^{13}$; portanto, esses fatores associados podem estar relacionados com a queda da PAM entre T2 e T3 dos animais 2 e 4 , justificando a administração de fármacos vasoativos para seu controle realizada neste experimento.

$\mathrm{Na}$ análise estatística de variância (ANOVA) dos dados, não foram verificadas diferenças estatisticamente significativas entre os tempos avaliados.

Tabela 1. Avaliação da pressão arterial média (PAM), em milímetros de mercúrio (mmHg), de cães submetidos a 30 minutos de CEC e uma hora de reperfusão pós-CEC

\begin{tabular}{lcccccc}
\hline & Animal 1 & Animal 2 & Animal 3 & Animal 4 & Média & DP \\
\hline T0 & 75 & 75 & 86 & 70 & 75.000 & 6.758 \\
T1 & 80 & 60 & 70 & 80 & 75.000 & 9.574 \\
T2 & 90 & 75 & 64 & 90 & 82.500 & 12.659 \\
T3 & 60 & 75 & 70 & 60 & 65.000 & 7.500 \\
Média & 77.500 & 75.000 & 70.000 & 75.000 & & \\
DP & 12.500 & 7.500 & 9.434 & 12.910 & & \\
\hline
\end{tabular}

DP: desvio padrão

Quanto à pressão venosa central (PVC), sua mensuração foi obtida através da colocação de um cateter venoso central na veia jugular, técnica que se mostrou de fácil execução e eficaz para o monitoramento hemodinâmico, administração de medicamentos e coleta de fluidos, como descrito também por outros autores ${ }^{14,15}$.

A PVC dos animais experimentais se manteve dentro dos valores de normalidade durante todos os tempos avaliados e com poucas oscilações (Tabela 2), pois esse fato está diretamente relacionado ao retorno venoso, à volemia e ao desempenho contrátil do ventrículo direito ${ }^{13}$; portanto, todos os animais estavam recebendo suporte adequado para a manutenção volêmica. $\mathrm{Na}$ análise estatística de variância (ANOVA) dos dados, não foram verificadas diferenças estatisticamente significativas entre os tempos avaliados. 
Tabela 2. Avaliação da pressão venosa central (PVC), em centímetros de água, de cães submetidos a 30 minutos de CEC e uma hora de reperfusão pós-CEC

\begin{tabular}{l|c|c|c|c|c|c}
\hline & Animal 1 & Animal 2 & Animal 3 & Animal 4 & Média & DP \\
\hline T0 & 5,5 & 4 & 6 & 4 & 4.000 & 1.155 \\
T1 & 6 & 5 & 5 & 9 & 5.500 & 1.893 \\
T2 & 5 & 5 & 9 & 9 & 7.000 & 2.309 \\
T3 & 5 & 4 & 11 & 4 & 4.500 & 3.366 \\
Média & 5.000 & 4.500 & 7.500 & 6.500 & & \\
DP & 0.577 & 0.577 & 2.754 & 2.887 & & \\
\hline
\end{tabular}

DP: desvio padrão

Considerando-se a saturação arterial de oxigênio $\left(\mathrm{SaO}_{2}\right)$, observou-se que se manteve com poucas oscilações durante todo $\mathrm{o}$ procedimento cirúrgico (Tabela 3), o que se justifica pelo fato de o animal ter sido mantido com suprimento de oxigênio $100 \%$ durante todos os tempos de avaliação do experimento, sendo fornecido através da sonda endotraqueal antes da CEC e suprido na própria máquina durante a CEC. No entanto, a $\mathrm{SaO}_{2}$ não pode ser considerada isoladamente para a avaliação da oxigenação real do animal, pois somente fornece a porcentagem de saturação da hemoglobina pelo oxigênio e não a oxigenação tecidual ${ }^{16}$. $\mathrm{Na}$ análise estatística de variância (ANOVA) dos dados, não foram verificadas diferenças estatisticamente significantes entre os tempos avaliados.

Tabela 3. Avaliação da oximetria através da saturação arterial de oxigênio $\left(\mathrm{SaO}_{2}\right)$, em porcentagem $(\%)$, de cães submetidos a 30 minutos de CEC e uma hora de reperfusão pós-CEC

\begin{tabular}{lcccccc}
\hline & Animal 1 & Animal 2 & Animal 3 & Animal 4 & Média & DP \\
\hline T0 & 99 & 100 & 99 & 100 & 99.500 & 0.577 \\
T1 & 99 & 97 & 99 & 95 & 97.500 & 1.9148 \\
T2 & 97 & 99 & 96 & 99 & 97.750 & 1.500 \\
T3 & 97 & 98 & 97 & 98 & 97.500 & 0,577 \\
Média & 98.000 & 98.500 & 97.750 & 98.000 & & \\
DP & 1.155 & 1.291 & 1.500 & 2.160 & & \\
\hline
\end{tabular}

DP: desvio padrão

Para a porcentagem de $\mathrm{CO}_{2}$ ao final da expiração $\left(\mathrm{ETCO}_{2}\right)$, que reflete de maneira muito próxima os valores de $\mathrm{PaCO}_{2}{ }^{17}$, as médias observadas estavam abaixo (animal 1) ou dentro do intervalo fisiológico (35 a $45 \mathrm{mmHg}$ ) para espécie ${ }^{18}$ durante o protocolo experimental (Tabela 4). Os valores que se mantiveram inferiores ao fisiológico foram provavelmente decorrentes de uma hiperventilação do animal, havendo aumento logo após o término da CEC, devido à interrupção da ventilação durante o procedimento, porém os valores mantiveram-se dentro da normalidade na maioria dos animais experimentais. Apesar da hipercapnia estar relacionada com o aparecimento de arritmias cardíacas $^{19}$, estas foram observadas neste estudo somente após a reversão da cardioplegia e com o retorno do batimento cardíaco, ao final da CEC, sendo corrigidas rapidamente. Na análise estatística de variância (ANOVA) dos dados, não foram verificadas diferenças estatisticamente significativas entre os tempos avaliados.

Tabela 4. Avaliação da capnografia pela medida do $\mathrm{CO}_{2}$ ao final da expiração $\left(\mathrm{ETCO}_{2}\right)$, em mmHg, de cães submetidos a 30 minutos de CEC e uma hora de reperfusão pós-CEC

\begin{tabular}{lcccccc}
\hline & Animal 1 & Animal 2 & Animal 3 & Animal 4 & Média & DP \\
\hline T0 & 43.1 & 43.1 & 24.0 & 42.4 & 42.750 & 9.439 \\
T1 & 38.2 & 38.2 & 34.8 & 60.1 & 38.200 & 11.628 \\
T2 & 22.0 & 42.8 & 48.2 & 47.0 & 44.900 & 12.221 \\
T3 & 12.2 & 40.9 & 47.2 & 46.4 & 43.650 & 16.555 \\
Média & 30.100 & 41.850 & 41.000 & 46.700 & & \\
DP & 14.313 & 2.255 & 11.456 & 7.692 & & \\
\hline
\end{tabular}

DP: desvio padrão 


\section{CONCLUSÃO}

A técnica de circulação extracorpórea é possível de ser realizada em cães, sem prejuízos hemodinâmicos graves, considerando-se os parâmetros avaliados neste experimento; contudo, ainda devem ser avaliados outros aspectos para que a técnica possa ser implementada na rotina cirúrgica veterinária.

\section{REFERÊNCIAS}

1. Freitas RR, Stopiglia AJ, Irino ET, Larsson MHMA. Tetralogia de Fallotem cão. Cienc. Rural. 2003; 33(6):1179-84.

2. Bureau S, Monnet E, Orton CE. Evaluation of survival rate and prognostic indicators for surgical treatment of leftto-right patent ductus arteriosus in dogs: 52 cases (19952003). J Am Vet Med Assoc. 2005; 11:227.

3. Freitas RR, Nogueira GAKA, Irino ET, Souza SL, Stopiglia AJ, Fantoni DT et al. Diagnosis and surgical correction of atrial septal defect by the inflow occlusion technique. Braz J Vet Res Anim Sci. 2005; 42(3):193-6.

4. Souza MHL, Elias DO. Fundamentos da Circulação Extracorpórea. 2nd ed. Rio de Janeiro: Centro Editorial Alfa Rio; 2006.

5. Dias RR, Silva IA, Fiorelli AI, Stolf NAG. Proteção cerebral: sítios de canulação arterial e vias de perfusão do cérebro. Rev Bras Cir Cardiovasc. 2007; 22(2)235-240.

6. Ribeiro SN, Monteiro GAS, Atallah FA, Amaral LG, Ferreira GS, Santana CL, Oliveira CJL, Oliveira ALA. Alterações ácido-básicas decorrentes da circulação extracorpórea em cães. Rev Bras Med Vet. 2014; 35(1):8490.

7. Barbosa NF, Cardinelli DM, Ercole FF. Determinantes de Complicações Neurológicas no Uso da Circulação Extracorpórea (CEC) Arq Bras Cardiol. 2010; 95(6):e151e157.

8. Monnet E, Orton C, Gaynor Js, Boon J, Wagner A, Linn $\mathrm{K}$, et al. Open resection for subvalvular aortic stenosis in dogs. J Am Vet Med Assoc. 1996; 209(7):1255-61.
9. Orton CE, Hellyer P, Hackett TB. Open surgical repair of tetralogy of Fallot in dogs. J Am Vet Med Assoc. 2001; 219(8):1089-93.

10. Martin JM, Orton CE, Boon JA, Mama KR, Gaynor JS, Brigth JM. Surgical correction of duble-chambered right ventricle in dogs. J Am Vet Med Assoc. 2002; 220(6):7704.

11. Orton CE, Hackett TB, Mama K, Boon JA. Technique and outcome of mitral valve replacement in dogs. J Am Vet Med Assoc. 2005; 226(9)1-4.

12. Tenorio SB, Cumino DO, Gomes DBG. Anestesia para o recém-nascido submetido a cirurgia cardíaca com circulação extracorpórea. Rev Bras Anestesiol. 2005; 55(1):118-34.

13. Alves FCG, Fiorelli AI. Monitorização hemodinâmica durante a circulação extracorpórea. Rev Latin Tec Extra. $2001 ; 8: 1$.

14. Portillo E, Mackin A, Hendrix PK, Boyle C, Chrestman L. Comparison of the modified Seldinger and through-theneedle jugular catheter placement techniques in the dog. $\mathrm{J}$ Vet Emerg Crit Care. 2006; 16:88-95.

15. Claude AK, Riedesel D, Riedesel EA. Electrocardiography-guided and retrospective analysis of central venous catheter placement in the dog. Vet Anaesth Analg. 2010; 37: 97-105.

16. Frey B, Shann F. Oxygen administration in infants. Arch Dis Child Feat Neon. 2003; 88:84-8, 2003.

17. Nishimori CT, Nunes N, Leite AV, Paula DP, Rezende ML, Souza AP, Santos PSP. Propofol ou sevofluorano sobre variáveis hemodinâmicas em cães submetidos à administração subaracnóidea de iohexol. Cienc Rural. 2005 ; 35(6):1345-1350.

18. Muir WW, Hubbell JAE. Manual de anestesia veterinária, 2nd ed. Porto Alegre: Artmed; 1997.

19. Lopes PCF, Nunes N, Sousa MG, Nishimori CTD, Carareto R, Paula DP, Santos PSP, Barbosa VF, Camacho AA. Variáveis fisiológicas e eletrocardiográficas de diferentes frações inspiradas de oxigênio em cães anestesiados com propofol. Rev Port Cienc Vet. 2008; 103(565-566):65-72. 